Research Article

\title{
Peristaltic Flow of a Nanofluid under the Effect of Hall Current and Porous Medium
}

\author{
Khalid Nowar \\ Department of Mathematics, Faculty of Science, King Saud University, P.O. Box 2455, Riyadh 11451, Saudi Arabia \\ Correspondence should be addressed to Khalid Nowar; khalidnowar@yahoo.com
}

Received 14 September 2013; Accepted 15 December 2013; Published 20 March 2014

Academic Editor: Mohamed Seddeek

Copyright (C) 2014 Khalid Nowar. This is an open access article distributed under the Creative Commons Attribution License, which permits unrestricted use, distribution, and reproduction in any medium, provided the original work is properly cited.

The problem of peristaltic flow of an incompressible viscous electrically conducting nanofluid in a vertical asymmetric channel through a porous medium is investigated by taking the Hall effects into account. The governing equations are formulated and simplified under the assumptions of long wavelength and low Reynolds number. The solutions for temperature and nanoparticle profiles are obtained by using the homotopy perturbation method (HPM) and closed form solutions for stream function and pressure gradient are developed. Finally, the effects of various emerging parameters on the physical quantities of interest are plotted and discussed.

\section{Introduction}

The peristaltic flow problems have attracted the attention of many researchers because of its increasing importance, specially in engineering, physiology, and biological systems. This is due to its many applications in real life such as urine transport from kidney to bladder through the ureter; transport of lymph in the lymphatic vessels; swallowing food through the esophagus; the movement of chyme in the gastrointestinal tract; ovum movement in the fallopian tube; vasomotion of small blood vessels such as venules, capillaries, and arterioles; and also in sanitary fluid transportation of corrosive fluids. Many modern mechanical devices have been designed on the principal of the peristaltic pumping for transporting fluids without internal moving parts, for example, the blood pump in the heart-lung machine and the peristaltic transport of noxious fluid in nuclear industry. For more details in this direction see, for example, [1-22].

The study of magnetohydrodynamics flows with Hall currents has important engineering applications in problems of magnetohydrodynamic generators and of Hall accelerators as well as in flight magnetohydrodynamics. Siddiqui et al. [23], studied effects of Hall current and heat transfer on MHD flow of a Burgers fluid due to a pull of eccentric rotating disks. Hall effects on peristaltic flow of a Maxwell fluid in a porous medium have been studied by Hayat et al. [24]. Hayat et al. [25], studied effects of Hall current and heat transfer on rotating flow of a second grade fluid through a porous medium. The effect of Hall currents on interaction of pulsatile and peristaltic transport induced flows of a particle fluid suspension that had been examined by Gad [26]. Recently the analytical solution for Hall and ion-slip effects on mixed convection flow of couple stress fluid between parallel disks is considered by Srinivasacharya and Kaladhar [27]. Narayana et al. [28] studied the effects of Hall current and radiation absorption on MHD micropolar fluid in a rotating system.

Nanofluid is a fluid containing nanometer-sized particles, called nanoparticles. These fluids are engineered colloidal suspensions of nanoparticles in a base fluid. The nanoparticles used in nanofluids are typically made of metals ( $\mathrm{Al}$, $\mathrm{Cu}$ ), oxides $\left(\mathrm{Al}_{2} \mathrm{O}_{3}\right)$, carbides $(\mathrm{SiC})$, nitrides (AlN, $\left.\mathrm{SiN}\right)$, or nonmetals (Graphite, carbon nanotubes) and the base fluid is usually a conductive fluid, such as water or ethylene glycol. Other base fluids are oil and other lubricants, biofluids, and polymer solutions. Nanoparticles range in diameter between 1 and $100 \mathrm{~nm}$. Nanofluids commonly contain up to a 5\% volume fraction of nanoparticles to ensure effective heat transfer enhancements. Nanofluids have novel properties that make them potentially useful in many applications in heat transfer, including microelectronics, fuel cells, pharmaceutical 
processes, and hybrid-powered engines. In engineering devices it has been widely used for engine cooling/vehicle thermal management, domestic refrigerator, chiller, heat exchanger, and nuclear reactor; in grinding, in machining, in space, defense, and ships; and in boiler flue gas temperature reduction.

Nowadays, there is a continuous focus of the researchers in the flow analysis of nanofluids because of, in addition to, the above mentioned applications these fluids enhance thermal conductivity of the base fluid enormously, which is beyond the explanation of any existing theory. They are also very stable and have no additional problems, such as sedimentation, erosion, and additional pressure drop. Choi [29] was the first who initiated this nanofluid technology. A detailed analysis of nanofluids was discussed by Buongiorno [30]. Khan and Pop [31] considered the boundary layer flow of a nanofluid passing a stretching sheet. Recently, Mustafa et al. [32] examined the influence of wall properties on the peristaltic flow of a nanofluid. The endoscopic effects on peristaltic flow of a nanofluid are introduced by Akbar and Nadeem [33]. The same authors Akbar and Nadeem studied the peristaltic flow of a PhanThien-Tanner nanofluid in a diverging tube [34]. Akbar et al. $[35,36]$ studied peristaltic flow of a nanofluid with slip effects and peristaltic flow of a nanofluid in a nonuniform tube.

To the best of the author's knowledge, no attempt is available in the literature which deals with the peristalsis of nanofluid including the Hall effect through a porous medium. Even the study of peristaltic flow of an electrically conducting nanofluid in a porous medium is not available. Also, the study of peristaltic flow of a nanofluid through a porous medium with Hall effects has not been made yet. The present study fills the gap in these directions.

The aim of this work is to investigate the peristaltic flow of an electrically conducting nanofluid in a vertical asymmetric channel through a porous medium with constant permeability. Modified Darcy's law for a nanofluid including the Hall current has been used for the modeling. In fact, the Hall effect is important when the Hall parameter is high. This happens when the magnetic field is strong. In most cases, the Hall term has been ignored in applying Ohm's law as it has no marked effects for small and moderate values of the magnetic field. However, the current trend in the application of magnetohydrodynamics is towards a strong magnetic field, so that the influence of electromagnetic force is noticeable. Under these conditions, the Hall current is important and it has marked effects on the magnitude and direction of the current density and consequently on the magnetic-force term. Therefore, it is important to study the effect of the Hall current on the flow. The governing equations of motion, energy, and nanoparticles for nanofluids have been reduced under the assumption of long wavelength and low Reynolds number. The reduced equations are then solved analytically via homotopy perturbation method [37-39]. The physical behaviors of emerging parameters are discussed through graphs. At the end the trapping phenomenon is discussed.

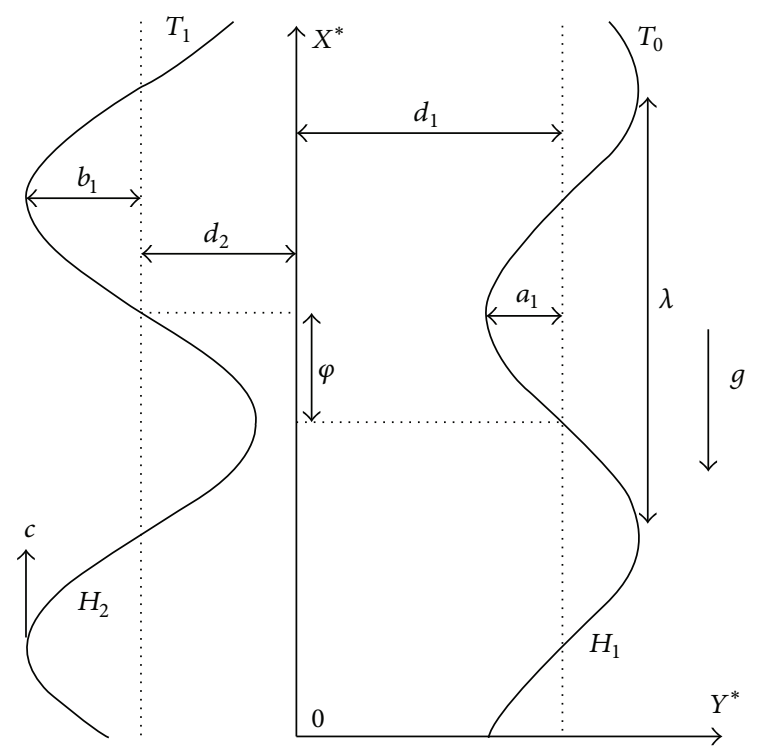

Figure 1: Geometry of the problem.

\section{Problem Formulation}

Consider the peristaltic flow of an incompressible viscous electrically conducting nanofluid in a two-dimensional vertical asymmetric channel of width $d_{1}+d_{2}$ through a porous medium. Asymmetry in the channel is produced by assuming the peristaltic wave trains propagating with constant speed $c$ along the walls $\left(H_{1}\right.$ is the right hand side wall and $H_{2}$ is the left hand side wall) to have different amplitudes and phases as shown in Figure 1. The shapes of the channel walls are represented as

$$
\begin{gathered}
Y=H_{1}(X, t)=d_{1}+a_{1} \cos \left(\frac{2 \pi}{\lambda}[X-c t]\right), \text { right wall, } \\
Y=H_{2}(X, t)=-d_{2}-b_{1} \cos \left(\frac{2 \pi}{\lambda}[X-c t]+\phi\right), \text { left wall, }
\end{gathered}
$$

where $a_{1}, b_{1}$ are the amplitudes of the right and left waves, $\lambda$ is the wave length, and $\phi$ is the phase difference. Further, $d_{1}$, $d_{2}, a_{1}, b_{1}$, and $\phi$ satisfy the following inequality:

$$
a_{1}^{2}+b_{1}^{2}+2 a_{1} b_{1} \cos \phi \leq\left(d_{1}+d_{2}\right)^{2},
$$

so that the walls will not intersect with each other. A uniform magnetic field with magnetic flux density vector $\mathbf{B}=\left(0,0, B_{0}\right)$ is applied; the induced magnetic field is neglected by taking a very small magnetic Reynolds number. The expression for the current density J including the Hall effect and neglecting ion-slip and thermoelectric effects is given by [40]

$$
\mathbf{J}=\sigma\left[\mathbf{E}+\mathbf{V} \wedge \mathbf{B}-\frac{1}{e n_{e}}(\mathbf{J} \wedge \mathbf{B})\right],
$$

where $\mathbf{J}$ is the current density, $\sigma$ is the electric conductivity of the fluid, $\mathbf{V}$ is the velocity vector, and $m=\sigma B_{0} / e n_{e}$ is the Hall parameter. It is also assumed that $\mathbf{E}=0$ (since there 




(a)

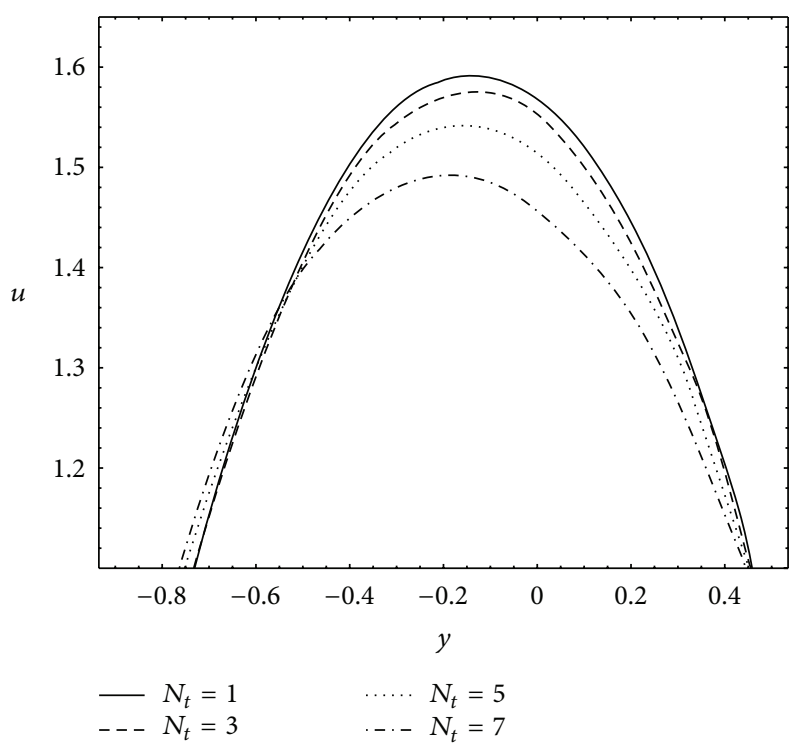

(c)
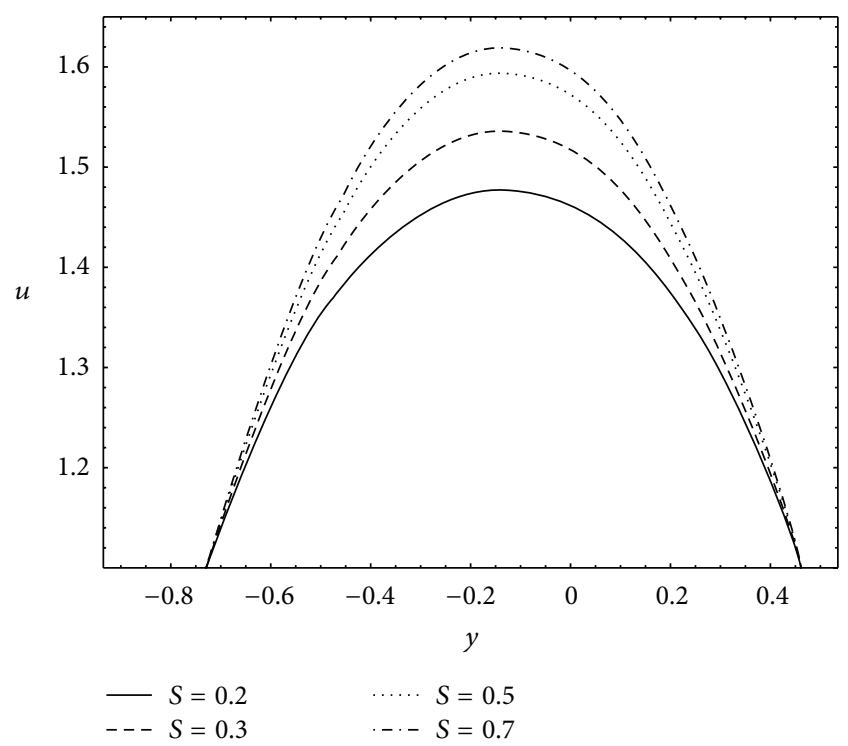

(b)



(d)

FIGURE 2: Variations of velocity profile $U$ with $y$ for various values of Hall parameter $m$ (panel a), porosity parameter $s$ (panel b), thermophoresis parameter $N_{t}$ (panel c), and local temperature Grashof number $G_{r}$ (panel d). The other parameters chosen are $F=2, x=0.1$, $d=1, M=1.2, a=0.1, b=0.5, N_{b}=0.8, N_{t}=0.5, s=0.5, \phi=0.2, G_{r}=0.5$, and $B_{r}=0.5$ (panel a); $F=2, x=0.1, d=1, M=1.2, a=0.1$, $b=0.5, N_{b}=0.8, N_{t}=0.5, m=1, \phi=0.2, G_{r}=0.5$, and $B_{r}=0.5$ (panel b); $F=2, x=0.1, d=1, M=1.2, a=0.1, b=0.5, N_{b}=0.8, m=1$, $s=0.5, \phi=0.2, G_{r}=0.5$ and $B_{r}=0.5$ (panel c); and $F=2, x=0.1, d=1, M=1.2, a=0.1, b=0.5, N_{b}=0.8, N_{t}=0.5, m=1, s=0.5$, $\phi=0.2$ and $B_{r}=0.5$ (panel d).

is no applied polarization voltage), $e$ is the electric charge and $n_{e}$ is the number density of electrons. The heat transfer and nanoparticle processes are maintained by considering temperatures $T_{0}, T_{1}$ and nanoparticle phenomena $C_{0}, C_{1}$ to the right and left sides walls of the channel, respectively. The fundamental equations governing this model [41] together with the generalized Ohm's law taking the effects of Hall currents and Maxwell's equations into account are

$$
\begin{gathered}
\rho_{f} \frac{d \mathbf{V}}{d t}=-\nabla P+\mu \nabla^{2} \mathbf{V}-\frac{\mu}{k_{1}} \mathbf{V}+\mathbf{J} \times \mathbf{B}+\mathbf{f}, \\
(\rho c)_{f} \frac{d T}{d t}=k \nabla^{2} T+(\rho c)_{p}\left[D_{B} \nabla C \nabla T+\left(\frac{D_{T}}{T_{0}}\right) \nabla T \nabla T\right] \\
\frac{d C}{d t}=D_{B} \nabla^{2} C+\left(\frac{D_{T}}{T_{0}}\right) \nabla^{2} T
\end{gathered}
$$






(a)

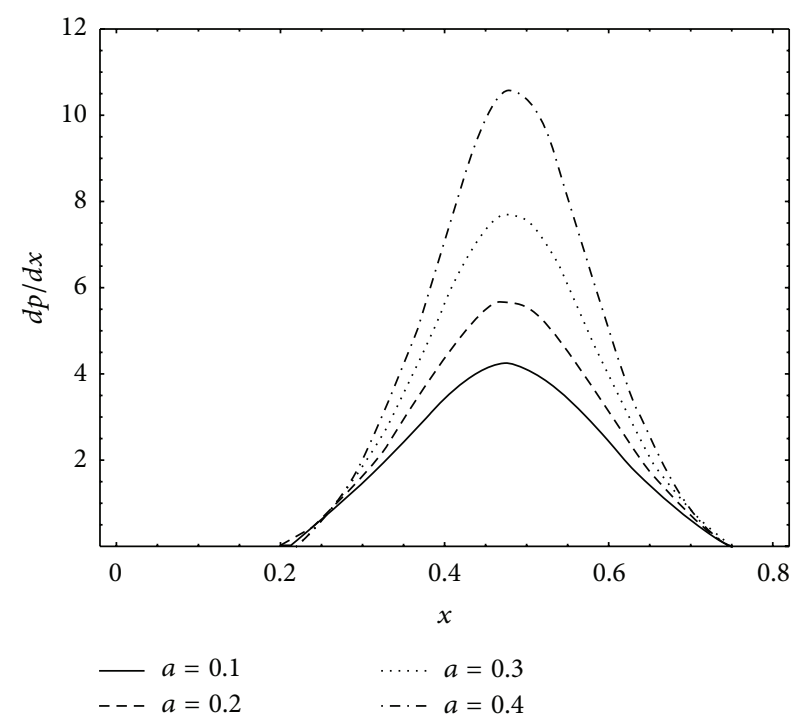

(c)

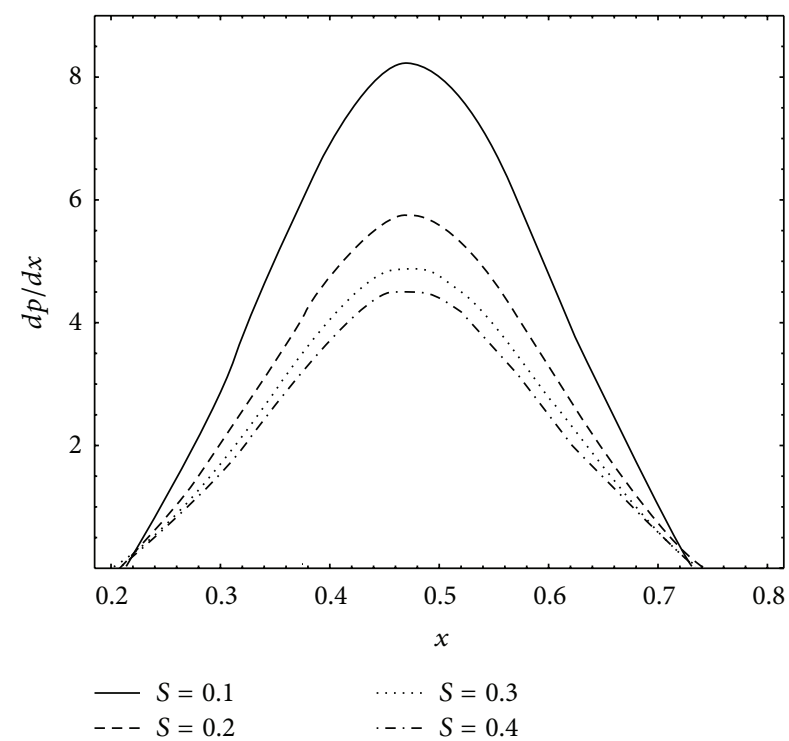

(b)

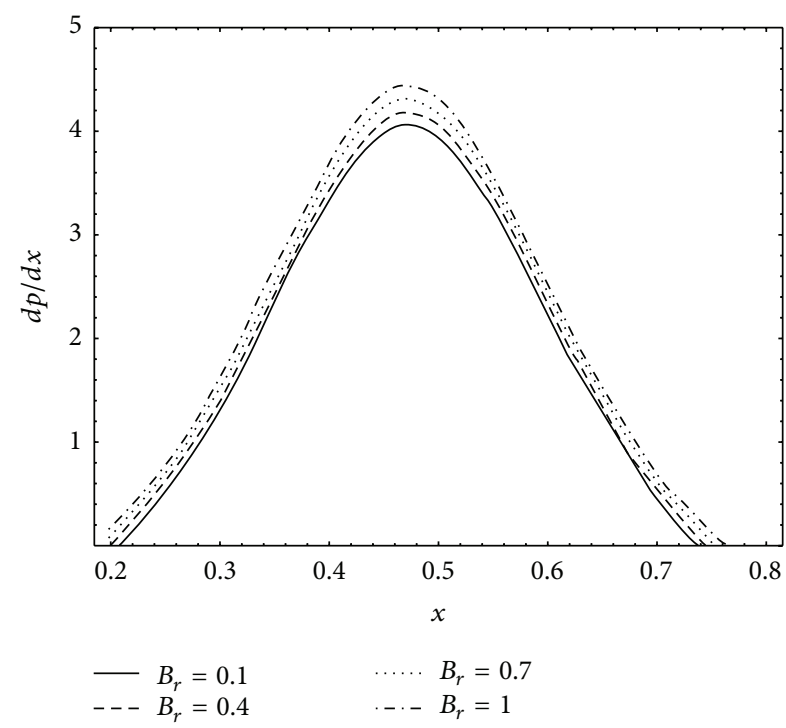

(d)

FIGURE 3: Variations of the axial pressure gradient $d p / d x$ with $x$ for various values of Hall parameter $m$ (panel a), porosity parameter $s$ (panel b), amplitude of right wave $a$ (panel c), and local nanoparticle Grashof number $B_{r}$ (panel d). The other parameters chosen are $F=-2, y=0.1$, $d=1, M=1.2, a=0.1, b=0.5, N_{b}=0.8, N_{t}=0.5, s=0.5, \phi=0.2, G_{r}=0.5$, and $B_{r}=0.5$ (panel a) $F=-2, y=0.1, d=1, M=1.2$, $a=0.1, b=0.5, N_{b}=0.8, N_{t}=0.5, m=1, \phi=0.2, G_{r}=0.5$, and $B_{r}=0.5$ (panel b); $F=-2, y=0.1, d=1, M=1.2, N_{t}=0.5, b=0.5$, $N_{b}=0.8, m=1, s=0.5, \phi=0.2, G_{r}=0.5$, and $B_{r}=0.5$ (panel c); and $F=-2, y=0.1, d=1, M=1.2, a=0.1, b=0.5, N_{b}=0.8, N_{t}=0.5$, $m=1, s=0.5, \phi=0.2$, and $G_{r}=0.5$ (panel d).

where $P$ is the pressure, $k_{1}$ is the permeability parameter, $\mu$ is the dynamic viscosity coefficient, $\mathbf{f}$ is the body forces, $\nabla^{2}$ is the Laplacian operator, $d / d t$ is the material derivative, $t$ is the time, $\rho_{f}$ is the density of the fluid, $\rho_{p}$ is the density of the particle, $T$ is the temperature of the fluid, $c$ is the volumetric volume expansion, $C$ is the nanoparticle phenomena, the ambient values of $T$ and $C$ as $y$ tends to $h_{1}$ are denoted by $T_{0}$ and $C_{0}, D_{B}$ is the Brownian diffusion coefficient, and $D_{T}$ is the thermophoretic diffusion coefficient.
The equations governing the two-dimensional motion of this model are

$$
\begin{gathered}
\frac{\partial U}{\partial X}+\frac{\partial V}{\partial Y}=0 \\
\rho_{f}\left(\frac{\partial U}{\partial t}+U \frac{\partial U}{\partial X}+V \frac{\partial U}{\partial Y}\right)
\end{gathered}
$$






(a)



(c)

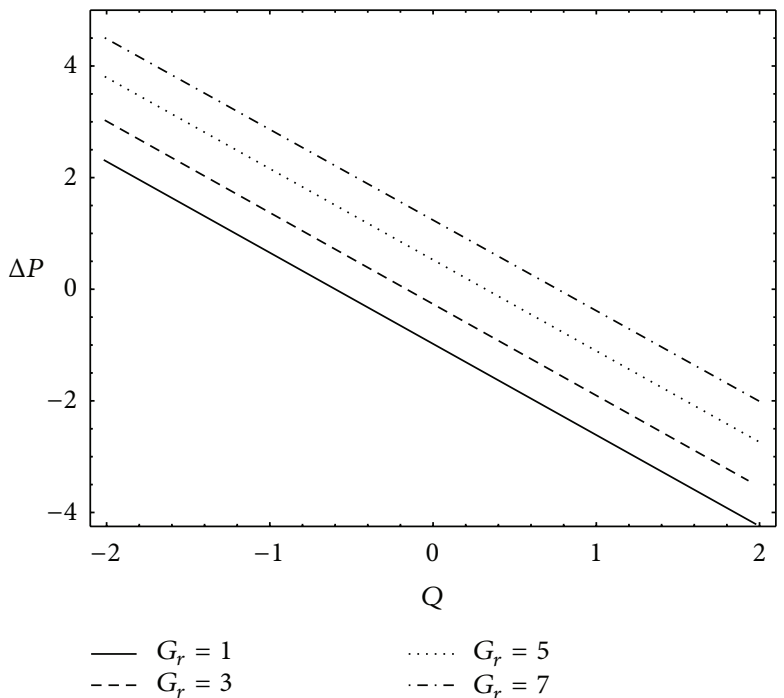

(b)

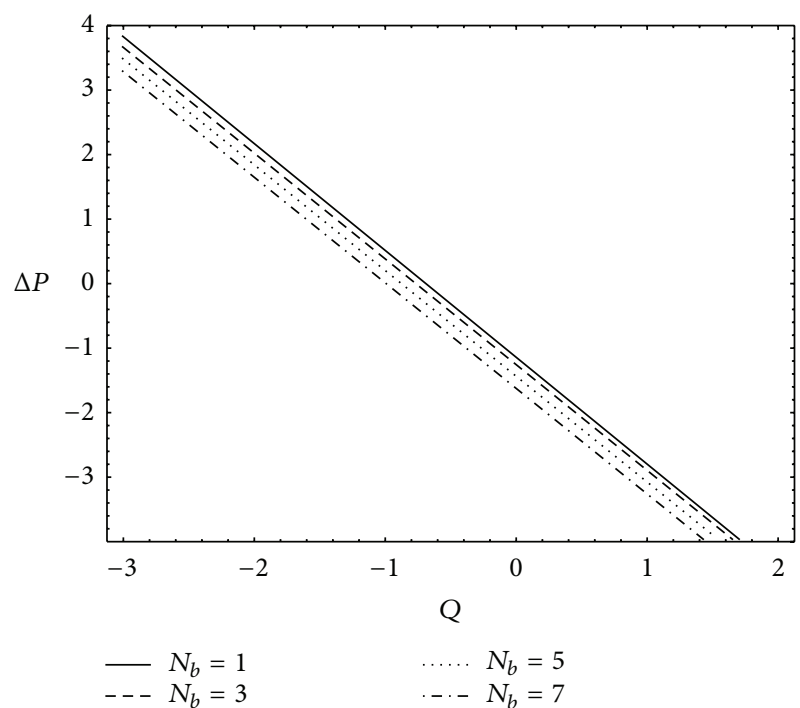

(d)

Figure 4: Variations of pressure rise $\Delta P$ versus flow rate $Q$ for different values of Hall parameter $m$ (panel a), local temperature Grashof number $G_{r}$ (panel b), porosity parameter $s$ (panel c), and the Brownian motion parameter $N_{b}$ (panel d). The other parameters chosen are $d=1, y=1, M=1.2, a=0.3, b=0.5, N_{b}=0.8, N_{t}=0.5, s=0.5, \phi=0.2, G_{r}=0.5$, and $B_{r}=0.5$ (panel a) $d=1, y=1, M=1.2, a=0.3$, $b=0.5, N_{b}=0.8, N_{t}=0.5, s=0.5, \phi=0.2, m=1$, and $B_{r}=0.5$ (panel b) $d=1, y=1, M=1.2, a=0.3, b=0.5, N_{b}=0.8, N_{t}=0.5, m=1$, $\phi=0.2, G_{r}=0.5$, and $B_{r}=0.5$ (panel c); and $d=1, y=1, M=1.2, a=0.3, b=0.5, m=1, N_{t}=0.5, s=0.5, \phi=0.2, G_{r}=0.5$, and $B_{r}=0.5$ (panel d).

$$
\begin{array}{cc}
=-\frac{\partial P}{\partial X}+\mu\left(\frac{\partial^{2} U}{\partial X^{2}}+\frac{\partial^{2} U}{\partial Y^{2}}\right) & \rho_{f}\left(\frac{\partial V}{\partial t}+U \frac{\partial V}{\partial X}+V \frac{\partial V}{\partial Y}\right) \\
-\frac{\mu}{k_{1}} U+\rho_{f} g \alpha\left(T-T_{0}\right) & =-\frac{\partial P}{\partial Y}+\mu\left(\frac{\partial^{2} V}{\partial X^{2}}+\frac{\partial^{2} V}{\partial Y^{2}}\right) \\
+\rho_{f} g \alpha\left(C-C_{0}\right)+\frac{\sigma B_{0}^{2}}{1+m^{2}}(m V-U), & -\frac{\mu}{k_{1}} V-\frac{\sigma B_{0}^{2}}{1+m^{2}}(m U+V),
\end{array}
$$




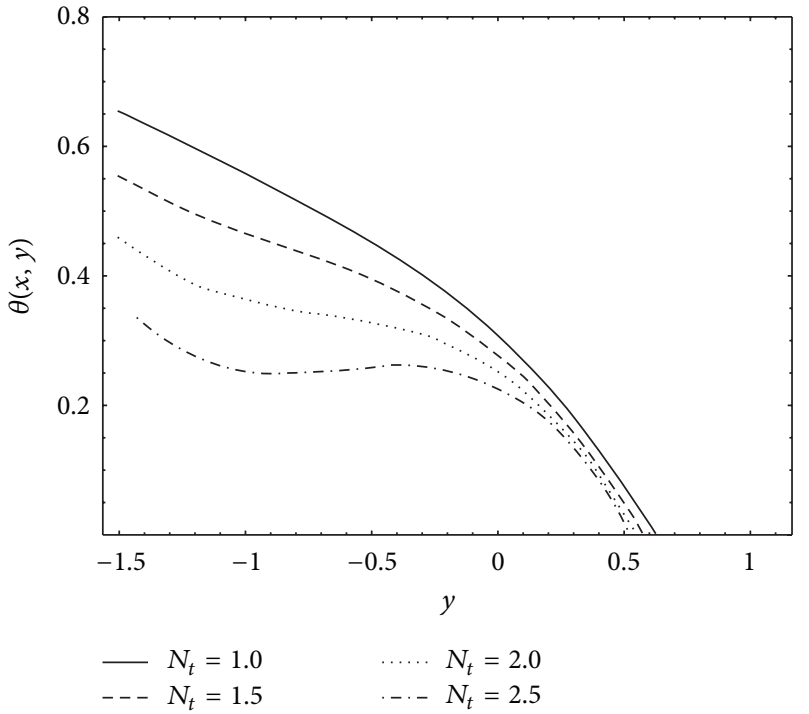

(a)



(b)

Figure 5: Variations of temperature profile $\Theta(x, y)$ with $y$ for different values of thermophoresis parameter $N_{t}$ (panel a) and the Brownian motion parameter $N_{b}$ (panel b). The other parameters chosen are $d=1, x=0.05, a=0.1, b=0.5, N_{b}=0.8$, and $\phi=0.2$ (panel a); $d=1$, $x=0.05, a=0.3, b=0.5, N_{t}=1.5$, and $\phi=0.2$ (panel b).

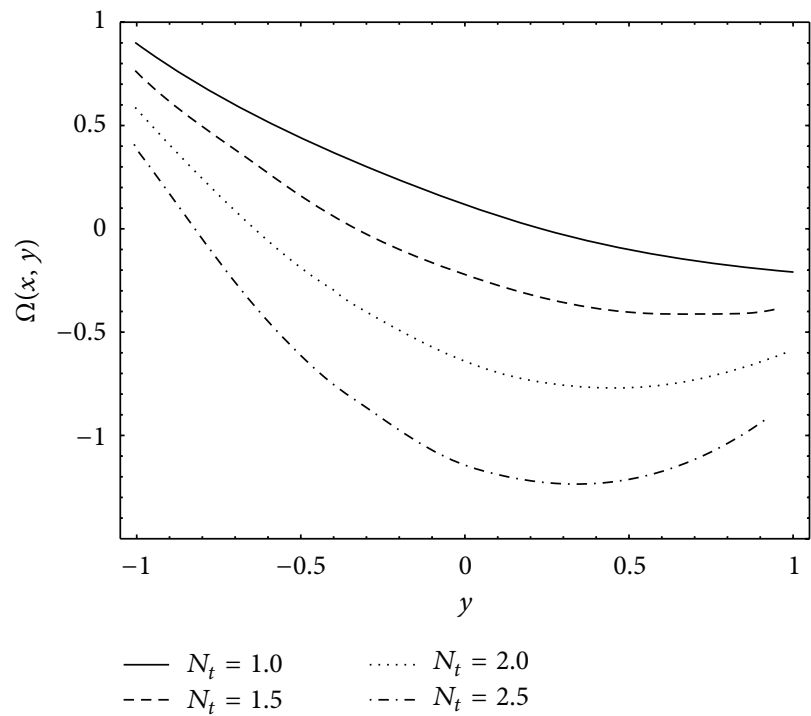

(a)

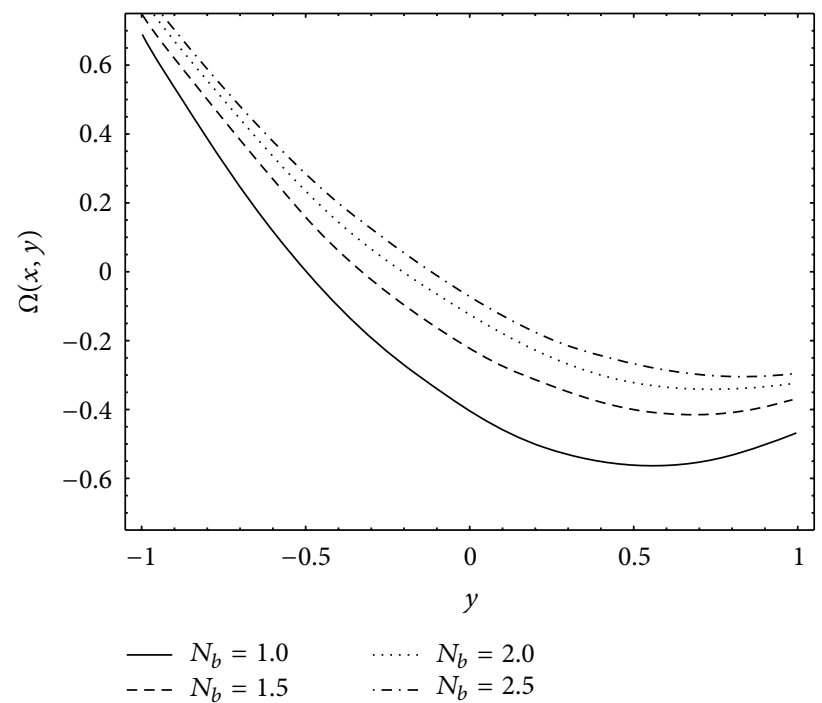

(b)

FIGURE 6: Variations of nanoparticle phenomena $\Omega(x, y)$ with $y$ for different values of thermophoresis parameter $N_{t}$ (panel a) and the Brownian motion parameter $N_{b}$ (panel b). The other parameters chosen are $d=1, x=0.25, a=0.3, b=0.5, N_{b}=1.5$, and $\phi=0.2$ (panel a); $d=1, x=0.25, a=0.3, b=0.5, N_{t}=1.5$, and $\phi=0.2$ (panel b).

$$
\begin{aligned}
\frac{\partial T}{\partial t}+ & U \frac{\partial T}{\partial X}+V \frac{\partial T}{\partial Y} \\
= & \left.\frac{k}{\rho c}\right)_{f}\left(\frac{\partial^{2} T}{\partial X^{2}}+\frac{\partial^{2} T}{\partial Y^{2}}\right)+\frac{(\rho c)_{p}}{(\rho c)_{f}} \\
& \times\left[D_{B}\left(\frac{\partial C}{\partial X} \frac{\partial T}{\partial X}+\frac{\partial C}{\partial Y} \frac{\partial T}{\partial Y}\right)\right. \\
& \left.+\frac{D_{T}}{T_{0}}\left(\left(\frac{\partial T}{\partial X}\right)^{2}+\left(\frac{\partial T}{\partial Y}\right)^{2}\right)\right],
\end{aligned}
$$

$$
\begin{aligned}
\frac{\partial C}{\partial t}+ & U \frac{\partial C}{\partial X}+V \frac{\partial C}{\partial Y} \\
& =D_{B}\left(\frac{\partial^{2} C}{\partial X^{2}}+\frac{\partial^{2} C}{\partial Y^{2}}\right)+\frac{D_{T}}{T_{0}}\left(\frac{\partial^{2} T}{\partial X^{2}}+\frac{\partial^{2} T}{\partial Y^{2}}\right)
\end{aligned}
$$

where $U$ and $V$ are the velocity components in the laboratory frame $(X, Y)$. Following Shapiro et al. [3] we introduce a wave frame of reference $(x, y)$ moving with velocity $c_{1}$ in which the motion becomes independent of time when the channel 


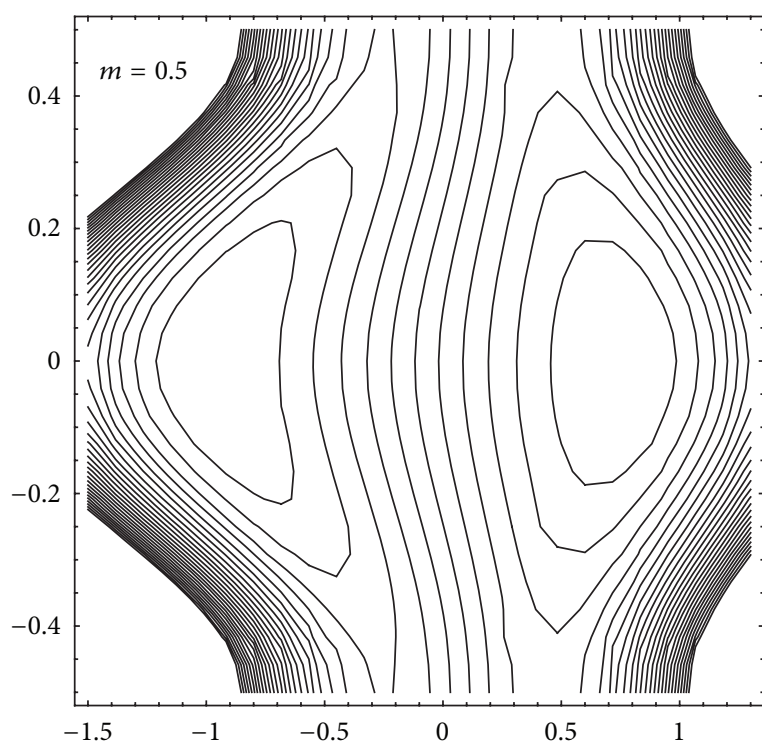

(a)

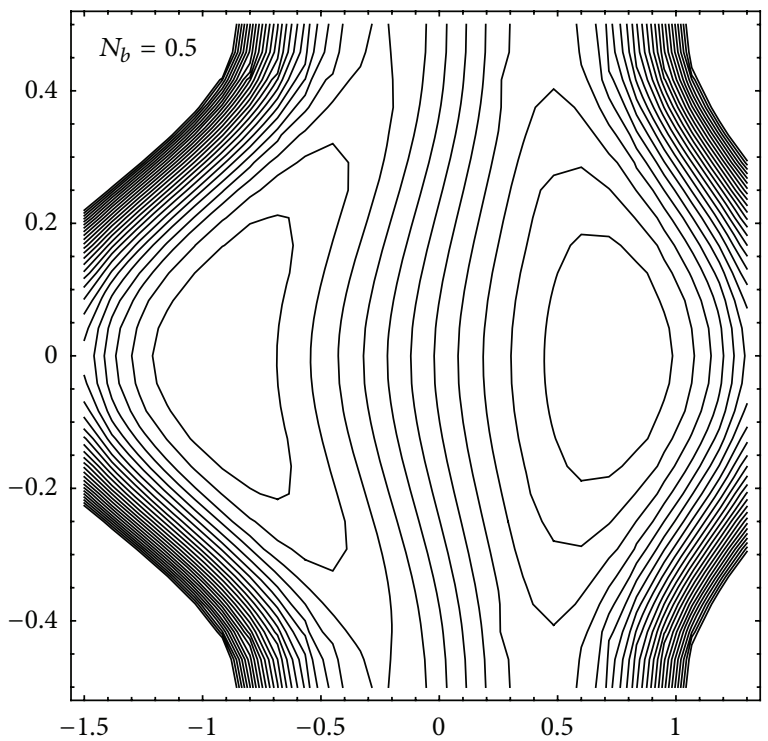

(c)

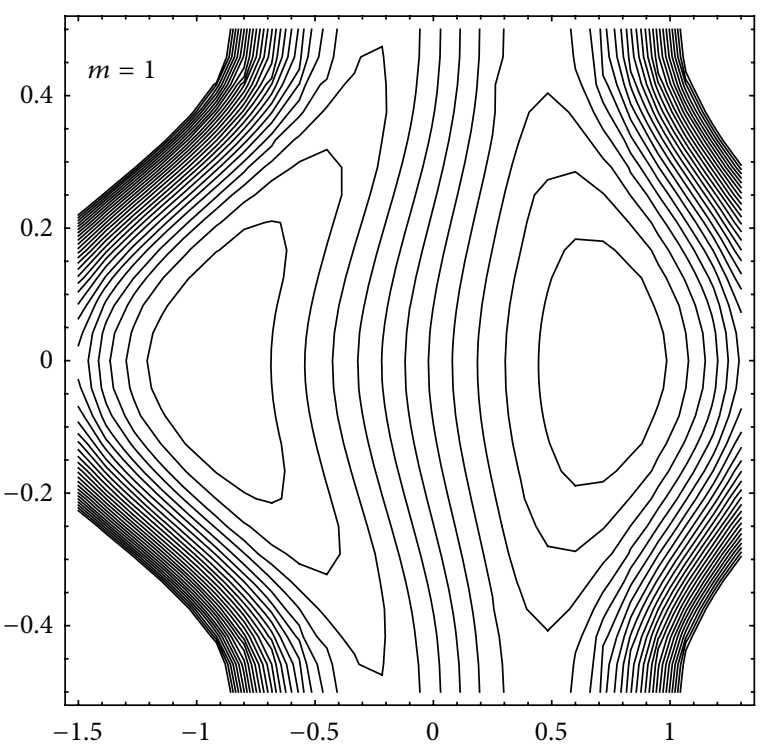

(b)

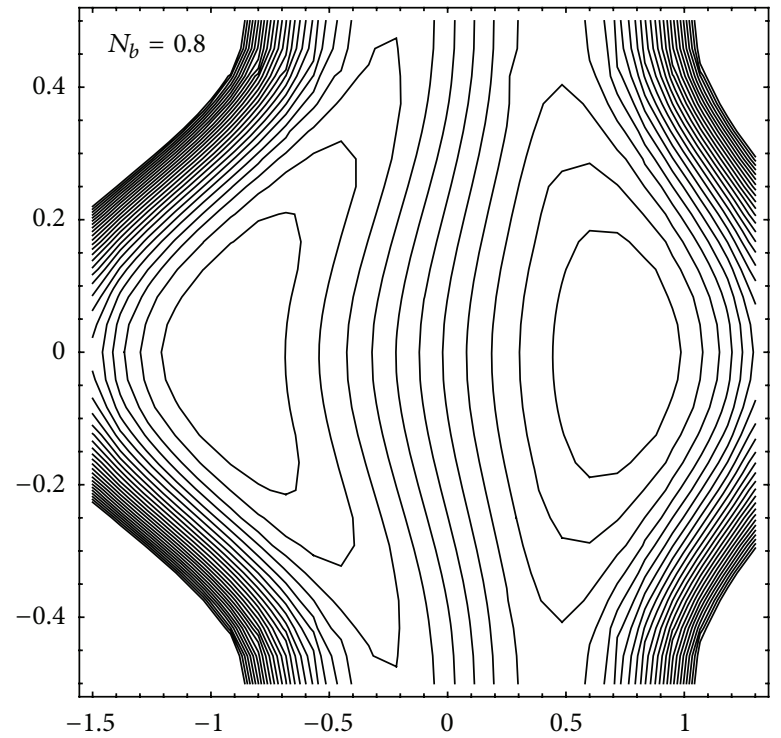

(d)

FiguRE 7: Streamlines for two different values of Hall parameter $m$ (panels $\mathrm{a}, \mathrm{b}$ ) and two different values of the Brownian motion parameter $N_{b}$ (panels c, d). The other parameters chosen are $Q=2, d=1, M=1.2, a=0.3, b=0.5, N_{b}=0.8, N_{t}=0.5, s=0.5, \phi=0.02, G_{r}=0.5$, and $B_{r}=0.5$ (panels a, b); $Q=2, d=1, M=1.2, a=0.3, b=0.5, m=1, N_{t}=0.5, s=0.5, \phi=0.02, G_{r}=0.5$, and $B_{r}=0.5$ (panels c, d).

is an integral multiple of the wavelength and the pressure difference at the the ends of the channel is a constant. The transformation from the laboratory frame of reference $(X, Y)$ to the wave frame of reference $(x, y)$ is given by

$$
\begin{gathered}
x=X-c_{1} t, \quad y=Y, \quad u=U-c_{1}, \\
v=V, \quad p(x)=P(x, t),
\end{gathered}
$$

where $(u, v), p$ and $(U, V), P$ are the velocity components and pressure in the wave and laboratory frames of reference, respectively. The pressure $p(x)$ remains a constant across any axial station of the channel under the assumption that the wavelength is large and the curvature effects are negligible.
The following are nondimensional variables:

$$
x^{*}=\frac{x}{\lambda}, \quad y^{*}=\frac{y}{d_{1}}, \quad u^{*}=\frac{u}{c_{1}}, \quad v^{*}=\frac{v}{c_{1}}
$$

$$
\begin{aligned}
& \delta=\frac{d_{1}}{\lambda}, \quad p^{*}=\frac{p d_{1}^{2}}{c_{1} \lambda \mu}, \quad t^{*}=\frac{t}{\lambda}, \\
& h_{1}=\frac{h_{1}}{d_{1}}, \quad h_{2}=\frac{h_{2}}{d_{1}}, \quad d=\frac{d_{2}}{d_{1}},
\end{aligned}
$$




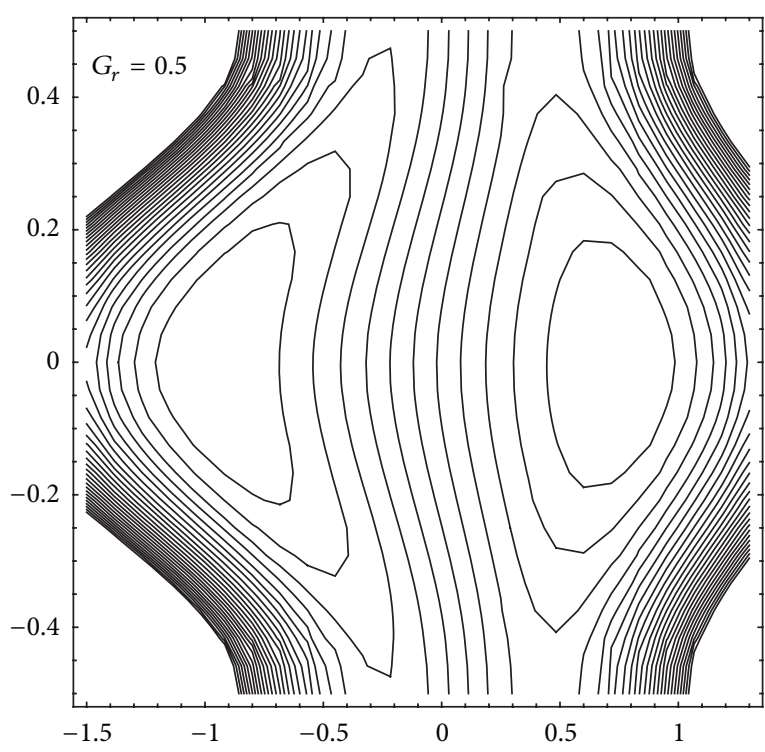

(a)

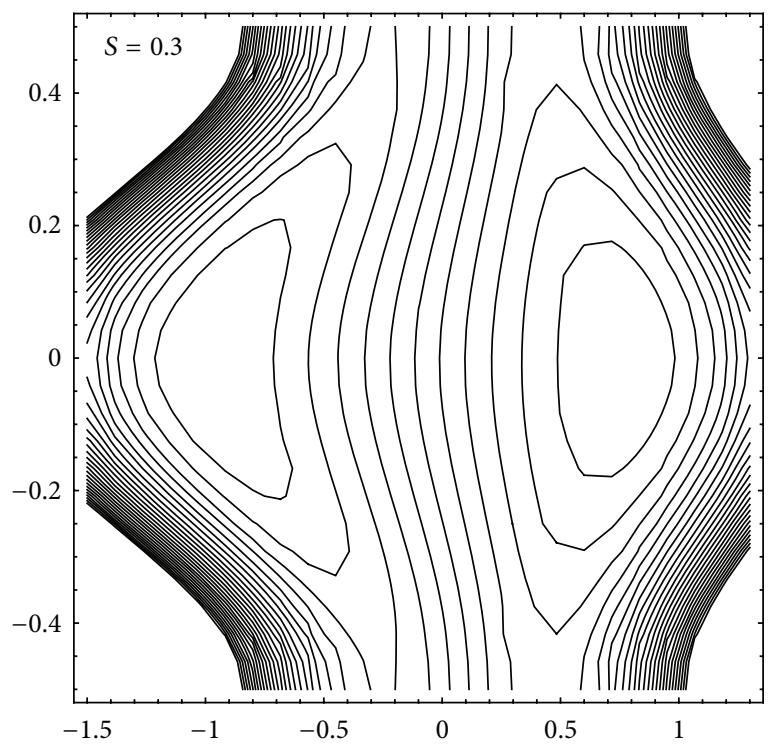

(c)

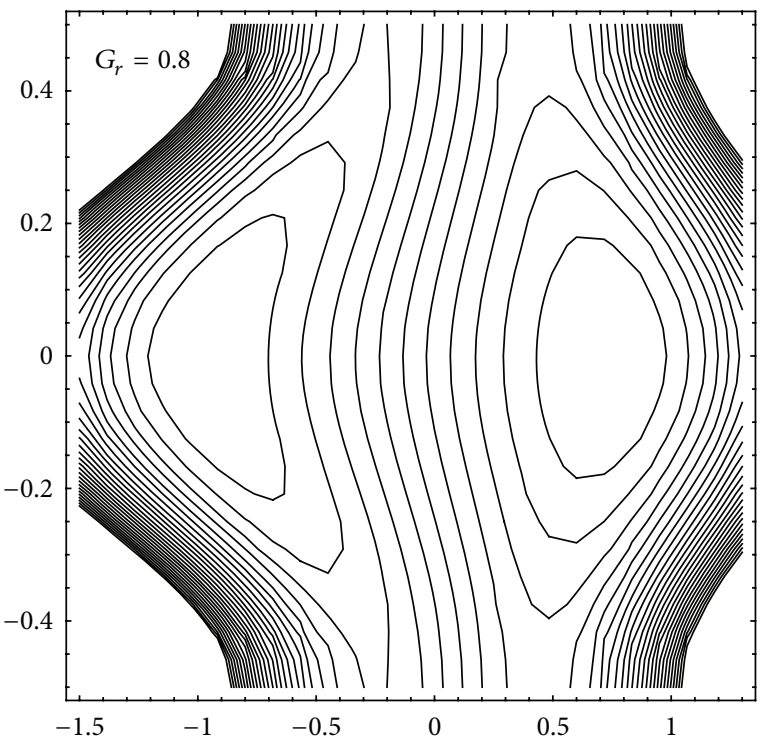

(b)

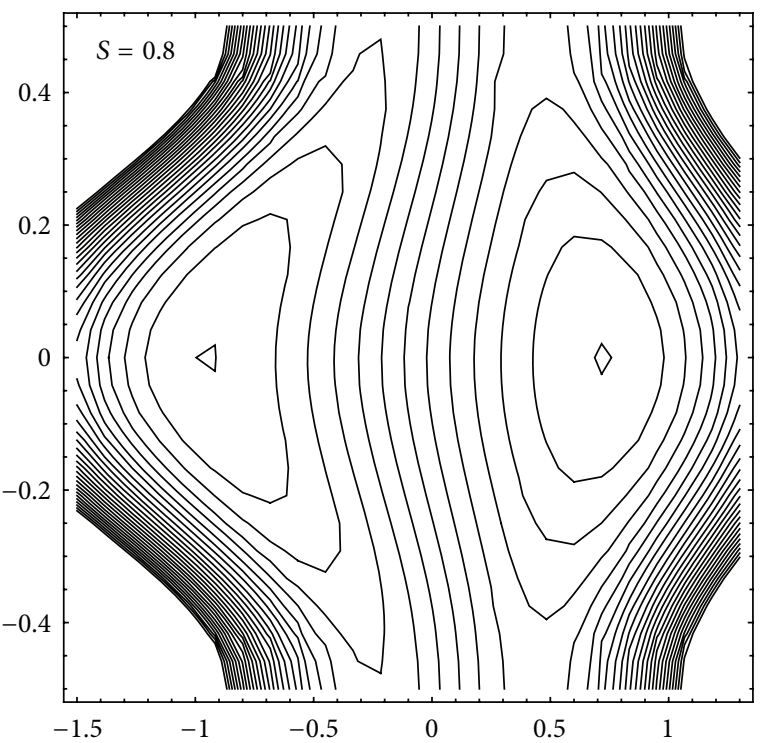

(d)

FIGURE 8: Streamlines for two different values of local temperature Grashof number $G_{r}$ (panels a, b) and two different values of porosity parameter $s$ (panels c, d). The other parameters chosen are $Q=2, d=1, M=1.2, a=0.3, b=0.5, N_{b}=0.8, N_{t}=0.5, s=0.5, \phi=0.02$, $m=1$, and $B_{r}=0.5$ (panels a, b); $Q=2, d=1, M=1.2, a=0.3, b=0.5, N_{b}=0.8, N_{t}=0.5, m=1, \phi=0.02, G_{r}=0.5$, and $B_{r}=0.5$ (panels $c, d)$.

$$
\begin{aligned}
& a=\frac{a_{1}}{d_{1}}, \quad b=\frac{b_{1}}{d_{1}}, \quad \operatorname{Re}=\frac{\rho_{f} d_{1} c_{1}}{\mu}, \quad s=\frac{k_{1}}{d_{1}^{2}}, \\
& \theta=\frac{T-T_{0}}{T_{1}-T_{0}}, \quad \Omega=\frac{C-C_{0}}{C_{1}-C_{0}}, \quad \alpha=\frac{k}{(\rho c)_{f}}, \\
& N_{b}=\frac{\rho c_{p} D_{B}\left(c_{1}-c_{0}\right)}{(\rho c)_{f} \alpha}, \quad \operatorname{Pr}=\frac{v}{\alpha},
\end{aligned}
$$$$
N_{t}=\frac{\rho c_{p} D_{T}\left(T_{1}-T_{0}\right)}{(\rho c)_{f} \alpha T_{0}}
$$$$
G_{r}=\frac{\alpha g\left(T_{1}-T_{0}\right) d_{1}^{2}}{\nu c_{1}}
$$$$
B_{r}=\frac{\alpha g\left(C_{1}-C_{0}\right) d_{1}^{2}}{\nu c_{1}}, \quad M=\frac{\sigma B_{0}^{2} d_{1}^{2}}{\mu}, \quad v=\frac{\mu}{\rho_{f}},
$$

where $\operatorname{Re}$ is the Reynolds number, $\delta$ is the dimensionless wave number, $s$ is the porosity parameter, $\mathrm{Pr}$ is the Prandtl number, $N_{b}$ is the Brownian motion parameter, $N_{t}$ is 
the thermophoresis parameter, $G_{r}$ is the local temperature Grashof number, $B_{r}$ is the local nanoparticle Grashof number, and $M$ is the magnetic parameter. Using (6) and (7) together with the stream function $\Psi$ defined by

$$
u=\frac{\partial \Psi}{\partial y}, \quad v=-\delta \frac{\partial \Psi}{\partial x}
$$

in (5), adopting the long wave length and low Reynolds number assumptions, we get the following:

$$
\begin{gathered}
\frac{\partial p}{\partial x}=\frac{\partial^{3} \Psi}{\partial y^{3}}-\Gamma^{2} \frac{\partial \Psi}{\partial y}+G_{r} \theta+B_{r} \Omega-\Gamma^{2} \\
\frac{\partial p}{\partial y}=0 \\
0=\frac{\partial^{2} \theta}{\partial y^{2}}+N_{b} \frac{\partial \theta}{\partial y} \frac{\partial \Omega}{\partial y}+N_{t}\left(\frac{\partial \theta}{\partial y}\right)^{2} \\
0=\frac{\partial^{2} \Omega}{\partial y^{2}}+\frac{N_{t}}{N_{b}} \frac{\partial^{2} \theta}{\partial y^{2}}
\end{gathered}
$$

Eliminating the pressure gradient between (9) and (10) we get

$$
\frac{\partial^{4} \Psi}{\partial y^{4}}-\Gamma^{2} \frac{\partial^{2} \Psi}{\partial y^{2}}+G_{r} \frac{\partial \theta}{\partial y}+B_{r} \frac{\partial \Omega}{\partial y}=0,
$$

where

$$
\Gamma^{2}=\left(\frac{1}{s}+\frac{M}{1+m^{2}}\right)
$$

The corresponding boundary conditions are

$$
\begin{aligned}
& \Psi=\frac{F}{2}, \quad \frac{\partial \Psi}{\partial y}=-1, \quad \theta=0, \quad \Omega=0 \quad \text { at } y=h_{1}, \\
& \Psi=-\frac{F}{2}, \quad \frac{\partial \Psi}{\partial y}=-1, \quad \theta=1, \quad \Omega=1 \quad \text { at } y=h_{2},
\end{aligned}
$$

where

$$
h_{1}=1+a \cos 2 \pi x, \quad h_{2}=-d-b \cos (2 \pi x+\phi),
$$

and $a, b, d$, and $\phi$ satisfy the relation

$$
a^{2}+b^{2}+2 a b \cos \phi \leq(1+d)^{2} \text {. }
$$

And, $F$ is the dimensionless average flux in the wave frame defined by

$$
F=\int_{h_{2}(x)}^{h_{1}(x)} \frac{\partial \Psi}{\partial y} d y=\Psi\left(h_{1}(x)\right)-\Psi\left(h_{2}(x)\right) .
$$

\section{Method of Solution}

3.1. Homotopy Perturbation Method. The homotopy perturbation method is a combination of the perturbation method and the homotopy method which eliminates the drawbacks of the traditional perturbation methods while keeping all their advantages. On the basis of the homotopy perturbation method [37-39] we can write (11) and (12) as follows:

$$
\begin{aligned}
H(q, \theta)= & (1-q)\left[L(\theta)-L\left(\theta_{10}\right)\right] \\
& +q\left[L(\theta)+N_{b} \frac{\partial \theta}{\partial y} \frac{\partial \Omega}{\partial y}+N_{t}\left(\frac{\partial \theta}{\partial y}\right)^{2}\right], \\
H(q, \Omega)= & (1-q)\left[L(\Omega)-L\left(\Omega_{10}\right)\right] \\
& +q\left[L(\Omega)+\frac{N_{t}}{N_{b}} \frac{\partial^{2} \theta}{\partial y^{2}}\right] .
\end{aligned}
$$

Or

$$
H(q, \theta)=L(\theta)-L\left(\theta_{10}\right)+q L\left(\theta_{10}\right)
$$$$
+q\left[N_{b} \frac{\partial \theta}{\partial y} \frac{\partial \Omega}{\partial y}+N_{t}\left(\frac{\partial \theta}{\partial y}\right)^{2}\right]
$$

$$
\begin{aligned}
H(q, \Omega)= & L(\Omega)-L\left(\Omega_{10}\right) \\
& +q L\left(\Omega_{10}\right)+q\left[\frac{N_{t}}{N_{b}} \frac{\partial^{2} \theta}{\partial y^{2}}\right],
\end{aligned}
$$

with $L=\partial^{2} / \partial y^{2}$ as the linear operator, the initial approximations $\theta_{10}$ and $\Omega_{10}$ can be defined as

$$
\theta_{10}(x, y)=\frac{y-h_{1}}{h_{1}-h_{2}}=\Omega_{10}(x, y)
$$

And assume that

$$
\begin{gathered}
\theta(y, q)=\theta_{0}+q \theta_{1}+q^{2} \theta_{2}+\cdots, \\
\Omega(y, q)=\Omega_{0}+q \Omega_{1}+q^{2} \Omega_{2}+\cdots .
\end{gathered}
$$

The solution of temperature and nanoparticle phenomenon (for $q=1$ ) are constructed by adopting the same procedure as done by [37-39]

$$
\begin{aligned}
\theta(x, y)= & \frac{A_{1}\left(N_{b}+2 N_{t}\right)}{6\left(h_{2}-h_{1}\right)} y^{3} \\
& -\frac{A_{1}\left[\left(N_{b}+2 N_{t}\right)\left(h_{1}+h_{2}\right)+2\left(h_{2}-h_{1}\right)\right]}{4\left(h_{2}-h_{1}\right)} y^{2}
\end{aligned}
$$




$$
\begin{gathered}
+\left(\left(A _ { 1 } \left[\left(N_{b}+2 N_{t}\right)\left(h_{1}^{2}+4 h_{1} h_{2}+h_{2}^{2}\right)\right.\right.\right. \\
\left.\left.+6\left(h_{2}^{2}-h_{1}^{2}\right)\right]+12\right) \\
\left.\times\left(12\left(h_{2}-h_{1}\right)\right)^{-1}\right) y \\
-\left(A_{1} h_{1} h_{2}\left[\left(N_{b}+2 N_{t}\right)\left(h_{1}+h_{2}\right)+6\left(h_{2}-h_{1}\right)\right]\right. \\
\left.+12 h_{1}\right)\left(12\left(h_{2}-h_{1}\right)\right)^{-1}, \\
\Omega(x, y)=\frac{A_{1} N_{t}}{2 N_{b}} y^{2}+\frac{2 N_{b}-A_{1} N_{t}\left(h_{2}^{2}-h_{1}^{2}\right)}{2 N_{b}\left(h_{2}-h_{1}\right)} y \\
+\frac{A_{1} N_{t} h_{1} h_{2}\left(h_{2}-h_{1}\right)-2 h_{1} N_{b}}{2 N_{b}\left(h_{2}-h_{1}\right)},
\end{gathered}
$$

where

$$
A_{1}=\frac{N_{b}+N_{t}}{\left(h_{2}-h_{1}\right)^{2}}
$$

Substituting (23) in (13), the exact solution for velocity is

$$
\begin{aligned}
\Psi(x, y)= & C_{11}+C_{12} y+C_{13} \cosh (\Gamma y) \\
& +C_{14} \sinh (\Gamma y)-\frac{A_{9}}{12 \Gamma^{2}} y^{4}-\frac{A_{10}}{6 \Gamma^{2}} y^{3}-\frac{A_{9}}{\Gamma^{4}} y^{2} \\
& -\left(\frac{A_{11}}{2 \Gamma^{2}}+\frac{A_{10}}{\Gamma^{4}}\right) y-\left(\frac{A_{11}}{\Gamma^{4}}+\frac{2 A_{9}}{\Gamma^{6}}\right) .
\end{aligned}
$$

And the pressure gradient is

$$
\begin{aligned}
\frac{\partial P}{\partial x}= & \left(A_{2} G_{r}+\frac{A_{9}}{3}\right) y^{3}+\left(A_{3} G_{r}+A_{6} B_{r}+\frac{A_{10}}{2}\right) y^{2} \\
& +\left(A_{4} G_{r}+A_{7} B_{r}\right) y \\
& +\left(A_{5} G_{r}+A_{8} B_{r}+\frac{A_{11}}{2}-\Gamma^{2}\left(C_{12}+1\right)\right) .
\end{aligned}
$$

The dimensionless pressure rise $\Delta P$ is given by

$$
\Delta P=\int_{0}^{1}\left(\frac{\partial P}{\partial x}\right) d x
$$

where

$$
\begin{gathered}
A_{2}=\frac{A_{1}\left(N_{b}+2 N_{t}\right)}{6\left(h_{2}-h_{1}\right)}, \\
A_{3}=-\frac{A_{1}\left[\left(N_{b}+2 N_{t}\right)\left(h_{1}+h_{2}\right)+2\left(h_{2}-h_{1}\right)\right]}{4\left(h_{2}-h_{1}\right)},
\end{gathered}
$$

$$
\begin{aligned}
& A_{4} \\
& =\frac{A_{1}\left[\left(N_{b}+2 N_{t}\right)\left(h_{1}^{2}+4 h_{1} h_{2}+h_{2}^{2}\right)+6\left(h_{2}^{2}-h_{1}^{2}\right)\right]+12}{12\left(h_{2}-h_{1}\right)},
\end{aligned}
$$

$A_{5}$

$$
=-\frac{A_{1} h_{1} h_{2}\left[\left(N_{b}+2 N_{t}\right)\left(h_{1}+h_{2}\right)+6\left(h_{2}-h_{1}\right)\right]+12 h_{1}}{12\left(h_{2}-h_{1}\right)},
$$

$$
A_{6}=\frac{N_{t}\left(N_{b}+N_{t}\right)}{2 N_{b}\left(h_{2}-h_{1}\right)^{2}}
$$

$$
A_{7}=\frac{2 N_{b}-N_{t} A_{1}\left(h_{2}^{2}-h_{1}^{2}\right)}{2 N_{b}\left(h_{2}-h_{1}\right)},
$$

$$
A_{8}=\frac{2 N_{t} A_{1} h_{1} h_{2}\left(h_{2}-h_{1}\right)-2 h_{1} N_{b}}{2 N_{b}\left(h_{2}-h_{1}\right)},
$$

$$
\begin{aligned}
A_{9}= & -G_{r}\left(A _ { 1 } \left[\left(N_{b}+2 N_{t}\right)\left(h_{1}^{2}+4 h_{1} h_{2}+h_{2}^{2}\right)\right.\right. \\
& \left.\left.+6\left(h_{2}^{2}-h_{1}^{2}\right)\right]+12\right) \\
& \times\left(4\left(h_{2}-h_{1}\right)\right)^{-1},
\end{aligned}
$$

$$
\begin{aligned}
A_{10}= & \frac{G_{r} A_{1}\left[\left(N_{b}+2 N_{t}\right)\left(h_{1}+h_{2}\right)+2\left(h_{2}-h_{1}\right)\right]}{2\left(h_{2}-h_{1}\right)} \\
& -\frac{B_{r} A_{1} N_{t}}{N_{b}}, \\
A_{11}= & -G_{r}\left(A _ { 1 } \left[\left(N_{b}+2 N_{t}\right)\left(h_{1}^{2}+4 h_{1} h_{2}+h_{2}^{2}\right)\right.\right. \\
& \left.\left.+6\left(h_{2}^{2}-h_{1}^{2}\right)\right]+12\right) \\
& \times\left(12\left(h_{2}-h_{1}\right)\right)^{-1} \\
& -\frac{B_{r}\left(2 N_{b}-N_{t} A_{1}\left(h_{2}^{2}-h_{1}^{2}\right)\right)}{2 N_{b}\left(h_{2}-h_{1}\right)},
\end{aligned}
$$


$C_{11}$

$=-\left(\operatorname{csch}\left(\frac{1}{2} \Gamma\left(h_{1}-h_{2}\right)\right)\right.$

$$
\begin{aligned}
\times\left(2 \left(6 \left(A _ { 1 1 } \left(2 \cosh \left(\Gamma\left(h_{1}-h_{2}\right)\right)\right.\right.\right.\right. \\
-\Gamma \sinh \left(\Gamma\left(h_{1}-h_{2}\right)\right) h_{1} \\
\left.+\Gamma \sinh \left(\Gamma\left(h_{1}-h_{2}\right)\right) h_{2}-2\right)
\end{aligned}
$$$$
+\Gamma\left(h_{1}+h_{2}\right)
$$$$
\times\left(3 \left(2 \cosh \left(\Gamma\left(h_{1}-h_{2}\right)\right)\right.\right.
$$$$
\left.+F \Gamma \sinh \left(\Gamma\left(h_{1}-h_{2}\right)\right)-2\right) \Gamma^{3}
$$$$
+A_{10}\left(\Gamma \left(\cosh \left(\Gamma\left(h_{1}-h_{2}\right)\right)\right.\right.
$$$$
+\Gamma \sinh \left(\Gamma\left(h_{1}-h_{2}\right)\right)
$$$$
\left.\times h_{2}+2\right) h_{1}^{2}
$$$$
-\left(\Gamma^{2} \sinh \left(\Gamma\left(h_{1}-h_{2}\right)\right) h_{2}^{2}\right.
$$$$
+2 \Gamma\left(2 \cosh \left(\Gamma\left(h_{1}-h_{2}\right)\right)\right.
$$$$
\text { +1) } h_{2}
$$$$
\left.+3 \sinh \left(\Gamma\left(h_{1}-h_{2}\right)\right)\right)
$$$$
\times h_{1}+h_{2}
$$$$
\times\left(3 \sinh \left(\Gamma\left(h_{1}-h_{2}\right)\right)+\Gamma\right.
$$$$
\times\left(\cosh \left(\Gamma\left(h_{1}-h_{2}\right)\right)+2\right)
$$$$
\left.\left.\left.\left.\times h_{2}\right)\right)\right)\right) \Gamma^{2}+A_{9}
$$

$$
\times\left(\Gamma^{4}\right.
$$$$
\times\left(\cosh \left(\Gamma\left(h_{1}-h_{2}\right)\right)\right.
$$$$
\left.+\Gamma \sinh \left(\Gamma\left(h_{1}-h_{2}\right)\right) h_{2}+3\right)
$$

$$
\times h_{1}^{4}-4 \Gamma^{3}\left(\sinh \left(\Gamma\left(h_{1}-h_{2}\right)\right)\right.
$$$$
+\Gamma\left(\cosh \left(\Gamma\left(h_{1}-h_{2}\right)\right) h_{2}\right)
$$$$
\times h_{1}^{3}+12 \Gamma^{2}
$$$$
\times\left(\cosh \left(\Gamma\left(h_{1}-h_{2}\right)\right)\right.
$$$$
+\Gamma \sinh \left(\Gamma\left(h_{1}-h_{2}\right)\right)
$$$$
\left.\times h_{2}+1\right)
$$$$
\times h_{1}^{2}-\Gamma
$$$$
\times\left(\Gamma^{4} \sinh \left(\Gamma\left(h_{1}-h_{2}\right)\right)\right.
$$$$
\times h_{2}^{4}+4 \Gamma^{3}
$$$$
\times \cosh \left(\Gamma\left(h_{1}-h_{2}\right)\right) h_{2}^{3}
$$$$
+12 \Gamma^{2}
$$

$$
\begin{aligned}
& \times \sinh \left(\Gamma\left(h_{1}-h_{2}\right)\right) \\
& \times h_{2}^{2}+48 \Gamma \\
& \times \cosh \left(\Gamma\left(h_{1}-h_{2}\right)\right) h_{2} \\
& \left.+48 \sinh \left(\Gamma\left(h_{1}-h_{2}\right)\right)\right) \\
& \times h_{1}+\Gamma^{4} \\
& \times\left(\cosh \left(\Gamma\left(h_{1}-h_{2}\right)\right)\right. \\
& +3) \\
& \times h_{2}^{4}+4 \Gamma^{3} \\
& \times \sinh \left(\Gamma\left(h_{1}-h_{2}\right)\right) \\
& \times h_{2}^{3}+24 \Gamma^{2} \\
& \times \cosh ^{2}\left(\frac{1}{2} \Gamma\left(h_{1}-h_{2}\right)\right) \\
& \times h_{2}^{2}+48 \\
& \times\left(\cosh \left(\Gamma\left(h_{1}-h_{2}\right)\right)\right. \\
& -1) \\
& +48 \Gamma \sinh \left(\Gamma\left(h_{1}-h_{2}\right)\right) \\
& \left.\left.\times h_{2}\right)\right) \text { ) } \\
& \times\left(2 4 \Gamma ^ { 6 } \left(-2 \sinh \left(\frac{1}{2} \Gamma\left(h_{1}-h_{2}\right)\right)\right.\right. \\
& +\Gamma \cosh \left(\frac{1}{2} \Gamma\left(h_{1}-h_{2}\right)\right) h_{1} \\
& \left.\left.-\Gamma \cosh \left(\frac{1}{2} \Gamma\left(h_{1}-h_{2}\right)\right) h_{2}\right)\right)^{-1}, \\
& C_{12}=-\left(-12 F \cosh \left(\frac{1}{2} \Gamma\left(h_{1}-h_{2}\right)\right) \Gamma^{5}\right. \\
& -24 \sinh \left(\frac{1}{2} \Gamma\left(h_{1}-h_{2}\right)\right) \Gamma^{4} \\
& -\cosh \left(\frac{1}{2} \Gamma\left(h_{1}-h_{2}\right)\right) A_{9} h_{1}^{4} \Gamma^{3} \\
& +\cosh \left(\frac{1}{2} \Gamma\left(h_{1}-h_{2}\right)\right) A_{9} h_{2}^{4} \Gamma^{3} \\
& +4 \sinh \left(\frac{1}{2} \Gamma\left(h_{1}-h_{2}\right)\right) \\
& \times A_{9} h_{1}^{3} \Gamma^{2}+4 \sinh \left(\frac{1}{2} \Gamma\left(h_{1}-h_{2}\right)\right) A_{9} h_{2}^{3} \Gamma^{2} \\
& -6 A_{11}\left(-2 \sinh \left(\frac{1}{2} \Gamma\left(h_{1}-h_{2}\right)\right)\right. \\
& +\Gamma \cosh \left(\frac{1}{2} \Gamma\left(h_{1}-h_{2}\right)\right) h_{1} \\
& \left.-\Gamma \cosh \left(\frac{1}{2} \Gamma\left(h_{1}-h_{2}\right)\right) h_{2}\right) \Gamma^{2}
\end{aligned}
$$




$$
\begin{aligned}
& -12 \cosh \left(\frac{1}{2} \Gamma\left(h_{1}-h_{2}\right)\right) \\
& \times A_{9} h_{1}^{2} \Gamma+12 \cosh \left(\frac{1}{2} \Gamma\left(h_{1}-h_{2}\right)\right) \\
& \times A_{9} h_{2}^{2} \Gamma+24 \sinh \left(\frac{1}{2} \Gamma\left(h_{1}-h_{2}\right)\right) \\
& \times A_{9} h_{1}+24 \sinh \left(\frac{1}{2} \Gamma\left(h_{1}-h_{2}\right)\right) A_{9} h_{2} \\
& +2 A_{10}\left(-\Gamma^{3} \cosh \left(\frac{1}{2} \Gamma\left(h_{1}-h_{2}\right)\right)\right. \\
& \times h_{1}^{3}+3 \Gamma^{2} \sinh \left(\frac{1}{2} \Gamma\left(h_{1}-h_{2}\right)\right) h_{1}^{2} \\
& -6 \Gamma \cosh \left(\frac{1}{2} \Gamma\left(h_{1}-h_{2}\right)\right) h_{1} \\
& +\Gamma^{3} \cosh \left(\frac{1}{2} \Gamma\left(h_{1}-h_{2}\right)\right) h_{2}^{3} \\
& +3 \Gamma^{2} \sinh \left(\frac{1}{2} \Gamma\left(h_{1}-h_{2}\right)\right) h_{2}^{2} \\
& +12 \sinh \left(\frac{1}{2} \Gamma\left(h_{1}-h_{2}\right)\right) \\
& \left.\left.+6 \Gamma \cosh \left(\frac{1}{2} \Gamma\left(h_{1}-h_{2}\right)\right) h_{2}\right)\right) \\
& \times\left(1 2 \Gamma ^ { 4 } \left(-2 \sinh \left(\frac{1}{2} \Gamma\left(h_{1}-h_{2}\right)\right)\right.\right. \\
& +\Gamma \cosh \left(\frac{1}{2} \Gamma\left(h_{1}-h_{2}\right)\right) h_{1} \\
& \left.\left.-\Gamma \cosh \left(\frac{1}{2} \Gamma\left(h_{1}-h_{2}\right)\right) h_{2}\right)\right)^{-1}, \\
& C_{13}=\left(\operatorname{csch}\left(\frac{1}{2} \Gamma\left(h_{1}-h_{2}\right)\right)\right. \\
& \times\left(12 F\left(\cosh \left(\Gamma h_{1}\right)-\cosh \left(\Gamma h_{2}\right)\right) \Gamma^{5}\right. \\
& +\left(\cosh \left(\Gamma h_{1}\right)+3 \cosh \left(\Gamma h_{2}\right)\right) A_{9} h_{1}^{4} \Gamma^{3} \\
& +\left(3 \cosh \left(\Gamma h_{1}\right)+\cosh \left(\Gamma h_{2}\right)\right) A_{9} h_{2}^{4} \Gamma^{3} \\
& +2\left(2\left(\sinh \left(\Gamma h_{1}\right)-\sinh \left(\Gamma h_{2}\right)\right) A_{9}+\Gamma\right. \\
& \left.\times\left(2 \cosh \left(\Gamma h_{1}\right)+\cosh \left(\Gamma h_{2}\right)\right) A_{10}\right) \\
& \times h_{2}^{3} \Gamma^{2}+2 h_{1}^{3} \\
& \times\left(\Gamma\left(\cosh \left(\Gamma h_{1}\right)+2 \cosh \left(\Gamma h_{2}\right)\right) A_{10}\right. \\
& -2 A_{9}\left(\sinh \left(\Gamma h_{1}\right)-\sinh \left(\Gamma h_{2}\right)\right. \\
& \left.\left.+\Gamma \cosh \left(\Gamma h_{2}\right) h_{2}\right)\right) \Gamma^{2} \\
& +6\left(2\left(\cosh \left(\Gamma h_{1}\right)+\cosh \left(\Gamma h_{2}\right)\right) A_{9}\right. \\
& \left.+\Gamma \sinh \left(\Gamma h_{1}\right)-\sinh \left(\Gamma h_{2}\right)\right) A_{10} \\
& \times h_{2}^{2} \Gamma-6 h_{1}^{2} \\
& \times\left(\Gamma A _ { 1 0 } \left(\sinh \left(\Gamma h_{1}\right)-\sinh \left(\Gamma h_{2}\right)\right.\right. \\
& \left.+\Gamma \cosh \left(\Gamma h_{2}\right) h_{2}\right) \\
& \left.-2\left(\cosh \left(\Gamma h_{1}\right)+\cosh \left(\Gamma h_{2}\right)\right) A_{9}\right) \\
& \times \Gamma-12
\end{aligned}
$$

$$
\begin{aligned}
& \times\left(\Gamma^{5}\left(\cosh \left(\Gamma h_{1}\right)-\cosh \left(\Gamma h_{2}\right)\right)\right. \\
& -2\left(\sinh \left(\Gamma h_{1}\right)-\sinh \left(\Gamma h_{2}\right)\right) \\
& \times A_{9} h_{2}+2 h_{1} \\
& \times\left(6\left(\cosh \left(\Gamma h_{1}\right)-\cosh \left(\Gamma h_{2}\right)\right) \Gamma^{5}\right. \\
& -3 \cosh \left(\Gamma h_{1}\right) A_{10} h_{2}^{2} \Gamma^{3}-2 A_{9} \\
& \times\left(\Gamma^{3} \cosh \left(\Gamma h_{1}\right) h_{2}^{3}+6 \Gamma\right. \\
& \times\left(\cosh \left(\Gamma h_{1}\right)+\cosh \left(\Gamma h_{2}\right)\right) \\
& \times h_{2} \\
& +6\left(\sinh \left(\Gamma h_{1}\right)\right. \\
& \left.\left.\left.\left.\left.-\sinh \left(\Gamma h_{2}\right)\right)\right)\right)\right)\right) \\
& \times\left(2 4 \Gamma ^ { 5 } \left(-2 \sinh \left(\frac{1}{2} \Gamma\left(h_{1}-h_{2}\right)\right)\right.\right. \\
& +\Gamma \cosh \left(\frac{1}{2} \Gamma\left(h_{1}-h_{2}\right)\right) h_{1} \\
& \left.\left.-\Gamma \cosh \left(\frac{1}{2} \Gamma\left(h_{1}-h_{2}\right)\right) h_{2}\right)\right)^{-1}, \\
& C_{14}=-\left(\operatorname{csch}\left(\frac{1}{2} \Gamma\left(h_{1}-h_{2}\right)\right)\right. \\
& \times\left(2 \left(-\cosh \left(\Gamma h_{1}\right)+\cosh \left(\Gamma h_{2}\right)\right.\right. \\
& \left.+\Gamma \sinh \left(\Gamma h_{2}\right) h_{1}-\Gamma \sinh \left(\Gamma h_{2}\right) h_{2}\right) \\
& \times\left(3 A_{10}\left(h_{1}^{2}-h_{2}^{2}\right) \Gamma^{2}+2 A_{9}\right. \\
& \left.\times\left(\Gamma^{2} h_{1}^{3}+6 h_{1}-h_{2}\left(\Gamma^{2} h_{2}^{2}+6\right)\right)\right) \\
& +\Gamma\left(\sinh \left(\Gamma h_{1}\right)-\sinh \left(\Gamma h_{2}\right)\right) \\
& \times\left(12 F \Gamma^{4}-12 h_{2} \Gamma^{4}+A_{9} h_{1}^{4} \Gamma^{2}\right. \\
& +3 A_{9} h_{2}^{4} \Gamma^{2}+2 A_{10} h_{1}^{3} \Gamma^{2} \\
& +4 A_{10} h_{2}^{3} \Gamma^{2} \\
& +12 A_{9} h_{1}^{2}+12 A_{9} h_{2}^{2}+2 h_{1} \\
& \times\left(6 \Gamma^{4}-3 A_{10} h_{2}^{2} \Gamma^{2}\right. \\
& \left.\left.\left.\left.-2 A_{9} h_{2}\left(\Gamma^{2} h_{2}^{2}+6\right)\right)\right)\right)\right) \\
& \times\left(2 4 \Gamma ^ { 5 } \left(-2 \sinh \left(\frac{1}{2} \Gamma\left(h_{1}-h_{2}\right)\right)\right.\right. \\
& +\Gamma \cosh \left(\frac{1}{2} \Gamma\left(h_{1}-h_{2}\right)\right) h_{1} \\
& \left.\left.-\Gamma \cosh \left(\frac{1}{2} \Gamma\left(h_{1}-h_{2}\right)\right) h_{2}\right)\right)^{-1} .
\end{aligned}
$$

When calculating the constants $C_{11}, C_{12}, C_{13}$, and $C_{14}$ we used the mathematica program. 


\section{Results and Discussion}

In this section we have studied the velocity, pressure gradient, pressure rise, temperature, and nanoparticle phenomena graphically. The effects of Hall parameter $m$, porosity parameter $s$, thermophoresis parameter $N_{t}$, and local temperature Grashof number $G_{r}$ on the velocity profile have been displayed in Figure 2. Figures 2(a) and 2(b) declare that velocity profile increases with an increase in the value of each of $m$ and $s$, Figure 2(c) announces that the velocity near the channel walls are not similar in view of $N_{t}$, and the velocity decreases by increasing $N_{t}$. In Figure 2(d) it is found that the velocity field increases in the region $y \in(-0.8,-0.16)$ and it decreases in the region $y \in(-0.16,0.45)$ with an increase in $G_{r}$. Figures 3(a) to 3(d) examine the pressure gradient for different values of Hall parameter $m$, porosity parameter $s$, amplitude of right wave $a$, and local nanoparticle Grashof number $B_{r}$. It is observed that the magnitude of pressure gradient decreases in view of an increase in $m$ and $s$ Figures 3(a) and 3(b). The situation is reversed in Figures $3(\mathrm{c})$ and 3(d), the magnitude of pressure gradient increases with an increase in $a$ and $B_{r}$. The pressure rise versus volume flow rate is shown in Figures 4(a) to 4(d). It is noticed from Figures 4(a) and 4(c) that in in the peristaltic pumping region $(\Delta P>0)$, the pressure rise increases with an increase in Hall parameter $m$ and porosity parameter $s$, while decreases in the augmented pumping region $(\Delta P<0)$. Free pumping region holds when $(\Delta P=0)$. It is also noticed that the pressure rise is an increasing function of the increase in local temperature Grashof number $G_{r}$ (Figure 4(b)); moreover, by increasing the value of Brownian motion parameter $N_{b}$, the pressure rise is a decreasing function (Figure 4(d)). Figures 5 and 6 demonstrate the variation of temperature profile and nanoparticle phenomena for different values of thermophoresis parameter $N_{t}$ and Brownian motion parameter $N_{b}$. Here the temperature profile decreases when $N_{t}$ and $N_{b}$ are increased and nanoparticle phenomenon decreases by increasing $N_{t}$, while it increases when $N_{b}$ is increased.

\section{Trapping Phenomenon}

The formation of an internally circulating bolus of fluid is called trapping. The trapped bolus moves along with the wave. The effects of Hall parameter $m$ and Brownian motion parameter $N_{b}$ on trapping can be observed through Figure 7, where the number of trapping bolus increases with an increase in $m$ and $N_{b}$ in the left half of the channel. Figure 8 depicts that increasing the local temperature Grashof number $G_{r}$ leads to a decrease in the number of trapping bolus in the left half of the channel but it increases in both the left and right halves of the channel with an increase in the porosity parameter $s$.

\section{Conclusions}

The effects of Hall current and porous media on peristaltic transport of a nanofluid are investigated. The main results are summarized as follows.
(1) Effects of Hall $m$ and porosity $s$ parameters are similar on velocity profile but thermophoresis parameter $N_{t}$ has opposite effect.

(2) The velocity near the channel walls is not similar in view of the local temperature Grashof number $G_{r}$.

(3) The magnitude of pressure gradient decreases by increasing Hall $m$ and porosity $s$ parameters. However it increases with an increase in amplitude of right wave $a$.

(4) The behaviors of Hall parameter $m$ and porosity parameter $s$ on the pressure rise are similar.

(5) The behaviors of local temperature Grashof number $G_{r}$ and Brownian motion parameter $N_{b}$ on the pressure rise are opposite.

(6) Temperature profile decreases when thermophoresis parameter $N_{t}$ and Brownian motion parameter $N_{b}$ are increased.

(7) Nanoparticle phenomenon decreases by increasing thermophoresis parameter $N_{t}$ while it increases by increasing Brownian motion parameter $N_{b}$.

(8) The number of trapping bolus increases in the left half of the channel due to an increase in Hall parameter $m$ and Brownian motion parameter $N_{b}$. On the other hand it decreases in the left half of the channel with an increase in local temperature Grashof number $G_{r}$.

(9) Increasing the magnitude of porosity parameter $s$ led to an increase in the number of trapping bolus in both halves of the channel.

\section{Conflict of Interests}

The author declares that there is no conflict of interests regarding the publication of this paper.

\section{Acknowledgment}

This project was supported by King Saud University, Deanship of Scientific Research, College of Science, Research center.

\section{References}

[1] T. W. Latham, Motion in a peristaltic pump [M.S. thesis], MITPress, Cambridge, Mass, USA, 1966.

[2] Y. C. Fung and C. S. Yih, "Peristaltic transport," Journal of Applied Mechanics, vol. 35, no. 4, pp. 669-675, 1968.

[3] A. H. Shapiro, M.Y. Jaffrin, and S. L. Weinberg, "Peristaltic pumoing with long wavelengths at low reynolds number," Journal of Fluid Mechanics, vol. 37, no. 4, pp. 799-825, 1969.

[4] L. M. Srivastava and V. P. Srivastava, "Peristaltic transport of blood: casson model. II," Journal of Biomechanics, vol. 17, no. 11, pp. 821-829, 1984.

[5] H. L. Agrawal and B. Anwaruddin, "Peristaltic ow of blood a branch," Ranchi University Mathematical Journal, vol. 15, pp. 111118,1984 . 
[6] E. F. El Shehawey and S. Z. A. Husseny, "Effects of porous boundaries on peristaltic transport through a porous medium," Acta Mechanica, vol. 143, no. 3, pp. 165-177, 2000.

[7] K. S. Mekheimer, "Peristaltic transport of a couple stress fluid in a uniform and non-uniform channels," Biorheology, vol. 39, no. 6, pp. 755-765, 2002.

[8] E. F. El-Shehawey and S. Z. A. Husseny, "Peristaltic transport of a magneto-fluid with porous boundaries," Applied Mathematics and Computation, vol. 129, no. 2-3, pp. 421-440, 2002.

[9] K. S. Mekheimer, "Non-linear peristaltic transport of magnetohydrodynamic flow in an inclined planar channel," Arabian Journal for Science and Engineering, vol. 28, no. 2 A, pp. 183-201, 2003.

[10] A. El Hakeem, A. El Naby, A. E. M. El Misiery, and I. I. El Shamy, "Hydromagnetic flow of fluid with variable viscosity in a uniform tube with peristalsis," Journal of Physics A, vol. 36, no. 31, pp. 8535-8547, 2003.

[11] M. Elshahed and A. M. H. Haroun, "Peristaltic transport of Johnson-Segalman fluid under effect of a magnetic field," Mathematical Problems in Engineering, vol. 2005, no. 6, pp. 663677, 2005.

[12] E. F. Elshehawey, N. T. Eldabe, E. M. Elghazy, and A. Ebaid, "Peristaltic transport in an asymmetric channel through a porous medium," Applied Mathematics and Computation, vol. 182, no. 1, pp. 140-150, 2006.

[13] T. Hayat, M. Javed, and N. Ali, "MHD peristaltic transport of a Jeffery fluid in a channel with compliant walls and porous space," Transport in Porous Media, vol. 74, no. 3, pp. 259-274, 2008.

[14] S. Srinivas and M. Kothandapani, "The influence of heat and mass transfer on MHD peristaltic flow through a porous space with compliant walls," Applied Mathematics and Computation, vol. 213, no. 1, pp. 197-208, 2009.

[15] S. Srinivas and R. Gayathri, "Peristaltic transport of a Newtonian fluid in a vertical asymmetric channel with heat transfer and porous medium," Applied Mathematics and Computation, vol. 215, no. 1, pp. 185-196, 2009.

[16] S. Nadeem and S. Akram, "Influence of inclined magnetic field on peristaltic flow of a Williamson fluid model in an inclined symmetric or asymmetric channel," Mathematical and Computer Modelling, vol. 52, no. 1-2, pp. 107-119, 2010.

[17] C. Vasudev, U. R. Rao, M. V. Subba Reddy, and G. P. Rao, "Peristaltic pumping of a Williamson uid through a porous medium in a horizontal channel with heat transfer," American Journal of Scientific and Industrial Research, vol. 1, no. 3, pp. 656666,2010

[18] T. Hayat, S. Hina, A. A. Hendi, and S. Asghar, "Effect of wall properties on the peristaltic flow of a third grade fluid in a curved channel with heat and mass transfer," International Journal of Heat and Mass Transfer, vol. 54, no. 23-24, pp. 51265136, 2011.

[19] K. Vajravelu, S. Sreenadh, K. Rajanikanth, and C. Lee, "Peristaltic transport of a Williamson fluid in asymmetric channels with permeable walls," Nonlinear Analysis: Real World Applications, vol. 13, no. 6, pp. 2804-2822, 2012.

[20] S. Hina, T. Hayat, S. Asghar, and A. A. Hendi, "Influence of compliant walls on peristaltic motion with heat/mass transfer and chemical reaction," International Journal of Heat and Mass Transfer, vol. 55, no. 13, pp. 3386-3394, 2012.

[21] S. Hina, T. Hayat, and A. Alsaedi, "Heat and mass transfer effects on the peristaltic flow of Johnson-Segalman fluid in a curved channel with compliant walls," International Journal of Heat and Mass Transfer, vol. 55, no. 13, pp. 3511-3521, 2012.

[22] T. Hayat, S. Noreen, M. S. Alhothuali, S. Asghar, and A. Alhomaidan, "Peristaltic flow under the effects of an induced magnetic field and heat and mass transfer," International Journal of Heat and Mass Transfer, vol. 55, no. 1-3, pp. 443-452, 2012.

[23] A. M. Siddiqui, M. A. Rana, and N. Ahmed, "Effects of hall current and heat transfer on MHD flow of a Burgers' fluid due to a pull of eccentric rotating disks," Communications in Nonlinear Science and Numerical Simulation, vol. 13, no. 8, pp. 1554-1570, 2008.

[24] T. Hayat, N. Ali, and S. Asghar, "Hall effects on peristaltic flow of a Maxwell fluid in a porous medium," Physics Letters A, vol. 363, no. 5-6, pp. 397-403, 2007.

[25] T. Hayat, Z. Abbas, and S. Asghar, "Effects of Hall current and heat transfer on rotating flow of a second grade fluid through a porous medium," Communications in Nonlinear Science and Numerical Simulation, vol. 13, no. 10, pp. 2177-2192, 2008.

[26] N. S. Gad, "Effect of Hall currents on interaction of pulsatile and peristaltic transport induced flows of a particle-fluid suspension," Applied Mathematics and Computation, vol. 217, no. 9, pp. 4313-4320, 2011.

[27] D. Srinivasacharya and K. Kaladhar, "Analytical solution for Hall and Ion-slip effects on mixed convection ow of couple stress uid between parallel disks," Mathematical and Computer Modelling, vol. 57, pp. 2494-2509, 2013.

[28] P. V. S. Narayana, B. Venkateswarlu, and S. Venkataramana, "Effects of Hall current and radiation absorption on MHD micropolar uid in a rotating system," Ain Shams Engineering Journal, vol. 4, no. 4, pp. 843-854, 2013.

[29] S. U. S. Choi, "Enhancing thermal conductivity of uids with nanoparticles," in Developments and Applications of NonNewtonian Ows, D. A. Siginer and H. P. Wang, Eds., vol. 66, pp. 99-105, ASME, New York, NY, USA, 1995.

[30] J. Buongiorno, "Convective transport in nanouids," ASME Journal of Heat Transfer, vol. 128, no. 3, pp. 240-250, 2006.

[31] W. A. Khan and I. Pop, "Boundary-layer ow of a nano uid past a stretching sheet," International Journal of Heat and Mass Transfer, vol. 53, no. 11-12, pp. 2477-2483, 2010.

[32] M. Mustafa, S. Hina, T. Hayat, and A. Alsaedi, "Inuence of wall properties on the peristaltic ow of a nanouid: analytic and numerical solutions," International Journal of Heat and Mass Transfer, vol. 55, no. 17-18, pp. 4871-4877, 2012.

[33] N. S. Akbar and S. Nadeem, "Endoscopic effects on peristaltic flow of a nanofluid," Communications in Theoretical Physics, vol. 56, no. 4, pp. 761-768, 2011.

[34] N. S. Akbar and S. Nadeem, "Peristaltic flow of a Phan-ThienTanner nanofluid in a diverging tube," Heat Transfer: Asian Research, vol. 41, no. 1, pp. 10-22, 2012.

[35] N. S. Akbar, S. Nadeem, T. Hayat, and A. A. Hendi, "Peristaltic flow of a nanofluid with slip effects," Meccanica, vol. 47, no. 5, pp. 1283-1294, 2012.

[36] N. S. Akbar, S. Nadeem, T. Hayat, and A. A. Hendi, "Peristaltic flow of a nanofluid in a non-uniform tube," Heat and Mass Transfer, vol. 48, no. 3, pp. 451-459, 2012.

[37] J.-H. He, "Approximate analytical solution for seepage flow with fractional derivatives in porous media," Computer Methods in Applied Mechanics and Engineering, vol. 167, no. 1-2, pp. 57-68, 1998.

[38] J.-H. He, "Homotopy perturbation technique," Computer Methods in Applied Mechanics and Engineering, vol. 178, no. 3-4, pp. 257-262, 1999. 
[39] J.-H. He, "Application of homotopy perturbation method to nonlinear wave equations," Chaos, Solitons and Fractals, vol. 26, no. 3, pp. 695-700, 2005.

[40] A. Bharali and A. K. Borkakati, "The effect of Hall currents on MHD flow and heat transfer between two parallel porous plates," Applied Scientific Research, vol. 39, no. 2, pp. 155-165, 1982.

[41] K. Khanafer, K. Vafai, and M. Lightstone, "Buoyancy-driven heat transfer enhancement in a two-dimensional enclosure utilizing nanofluids," International Journal of Heat and Mass Transfer, vol. 46, no. 19, pp. 3639-3653, 2003. 


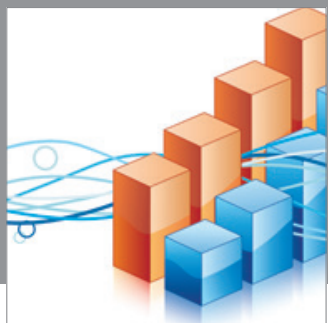

Advances in

Operations Research

mansans



The Scientific World Journal



Submit your manuscripts at http://www.hindawi.com
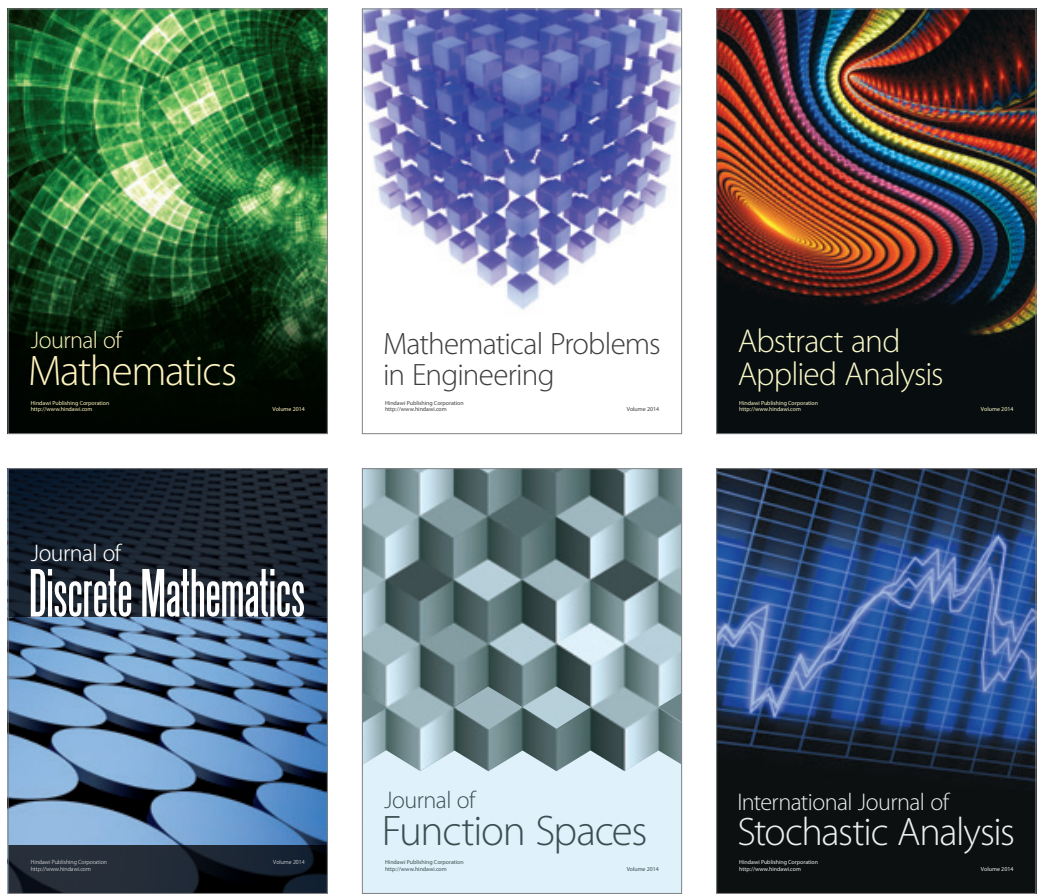

Journal of

Function Spaces

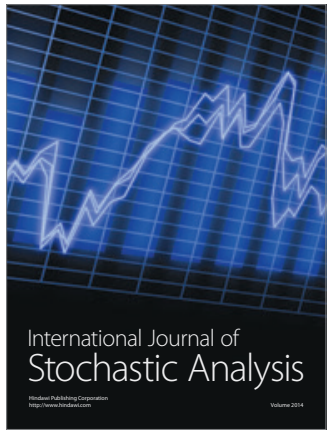

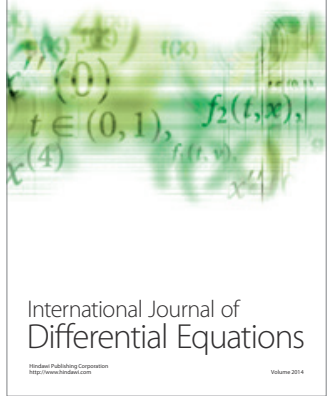
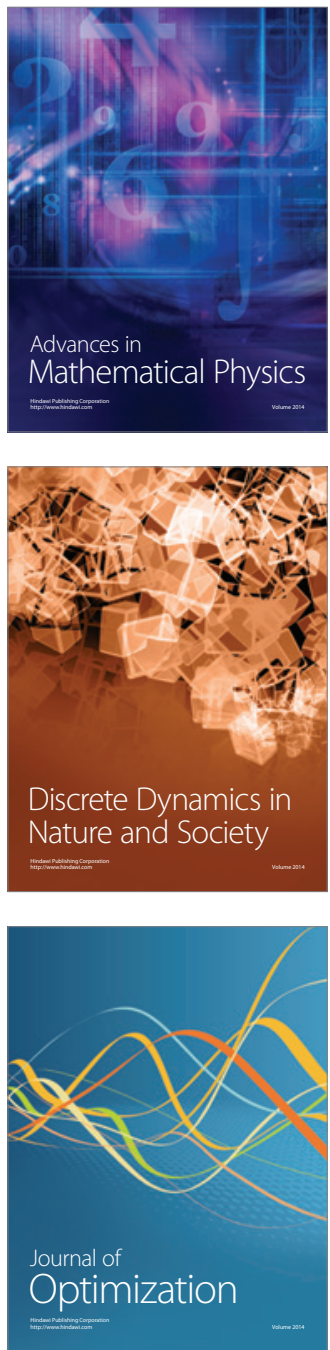